\title{
FEATHERING CRACKS FORMATION AT SINGLE WING CRACK EVOLUTION
}

\author{
A.V. Chentsov*, N.M. Osipenko**
}

\begin{abstract}
Wing crack formation was studied in a compression test system on PMMA and gypsum. Wing cracks are the tensile cracks starting in the area close to the top of the preexisting defect-an inclined initial cut. The crack growth is followed by relative shear motion between the surfaces of the initial defect. The shear zone was studied separately and a set of feathering cracks developed. The scenarios of complex wingcrack systems development are discussed.
\end{abstract}

Keywords: Wing Crack, Shear Fracture, Secondary Cracks.

\section{Introduction}

The interest to the process of quasi-brittle fracture at compression in structured materials has led to several models in which various sources of primary faults were considered. The extensive experimental and theoretical studies of fracture scenarios were performed to explain the initiation and propagation of cracks in samples of rocks, ice and other brittle materials at compression. The aim of the paper is experimental evaluation of the scenarios of secondary fracture development at compression of a body containing the inclined plane of shear (or a narrow cavity). The most fully studied are the situations in which fracture occurred in the neighborhood of initial defects in the form of rectilinear cuts inclined relative to the axis of compression. Let us consider this situation.

Surfaces of the cuts can be either separated, or contacting. In the latter case they interact with friction and slipping. A set of secondary cracks that surround the shear crack and form a shear band so that the shear reminds a bird's feather (as in Fig. 1) is usually called feathering cracks. The arrows in Fig. 1 show the crack growth direction.

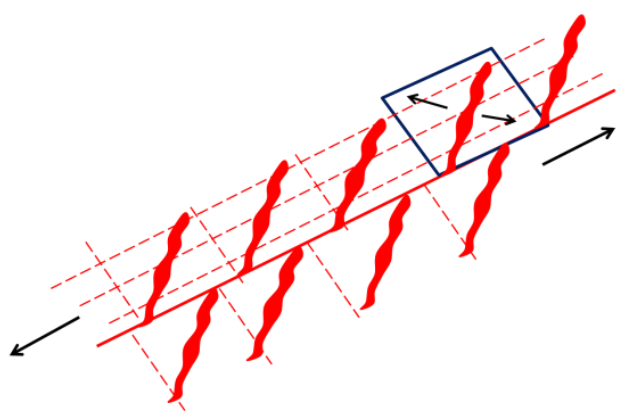

Fig. 1: The schematic of feathering cracks formation on the sides of initial shear plane

Senior Researcher Alexander V. Chentsov, PhD.: Ishlinsky Institute for Problems in Mechanics of the Russian Academy of Sciences, Prospekt Vernadskogo, 101-1, Moscow 119526; Russia, chentsov@ipmnet.ru

** Senior Researcher Nikolai M. Osipenko, PhD.: Ishlinsky Institute for Problems in Mechanics of the Russian Academy of Sciences, Prospekt Vernadskogo, 101-1, Moscow 119526; Russia, osipnm@mail.ru 
Compression of a rock sample with a cut is usually followed by formation of two types of cracks in the neighborhood of the cut: the feathering cracks and secondary cracks generated usually after initiation of primary feathering. The feathering may be limited to the wing cracks - the tensile cracks starting in the area close to the top of the initial cut. In this case crack growth is followed by relative shear motion between the top and lower surfaces of the initial defect.

The wing crack fracture model is one of the most widely used models used for explanation of brittle fracture of the structured materials at compression (Moss, 1982, Germanovich, 1994 and 2000). Wing cracks consisting of initial plane origin and a feathering extend in the steady mode along the direction of the maximum compression if secondary cracks are tensile cracks (Zavattieri, 2011).

The fracture in the form of a combination of local shear and wing-cracks provoked by it, observed in various materials and fracture structures of various scales are common structures of brittle fracture and mechanism of stage-by-stage brittle fracture of the structured (porous and cracked) media. At the same time, development of the mechanical - mathematical models of such structures is just at the beginning. In particular, mechanisms and scenarios of secondary cracks and shear feathering structures development need specification, including the interaction of the largest elements of structure. Beside the cases when main contribution is created by single wing cracks, the fracture scenarios with participation of secondary structures are of special importance.

\section{Methods}

Wing crack formation was studied in a "Testsystems" compression test system on PMMA and gypsum rectangular samples. The general view of the compression test system is given in Fig. 2. The sample was placed on a stable lower plate. Upper part is equipped with a special device that can measure shear forces and lateral displacement in horizontal plane, acting during compression. So the upper compressive plate is mobile if the specimen cracks, bends or slips.

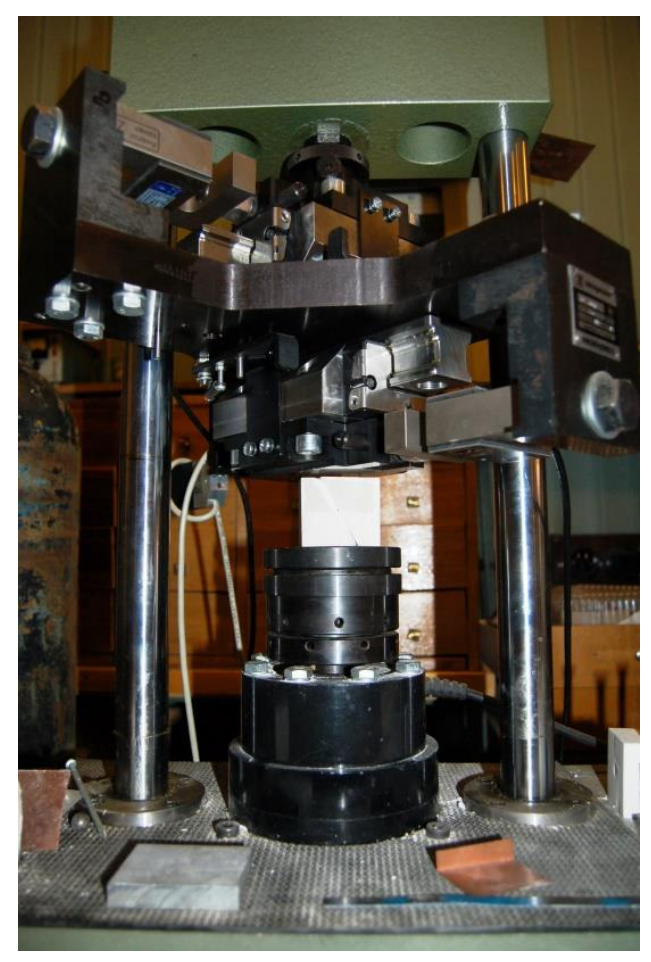

Fig. 2:Overall view of the compression test system. A rectangular gypsum sample is placed on a stable lower plate 


\section{Wing-crack experimental modeling}

Experiments on estimation of wing crack formation conditions were made on rectangular plane PMMA samples. Prior to axial loading of the sample, the initial cut was made at an angle to the loading axis through a hole. The samples were then compressed along the longer dimension up to occurrence of wing cracks. The final state of one sample is shown in Fig. 3a (sample dimensions (WxHxT): 42x58x12 mm, cut inclination 60 degrees, cut thickness $0.9 \mathrm{~mm}$, hole diameter $3 \mathrm{~mm}$, after $45 \mathrm{kN}$ load).
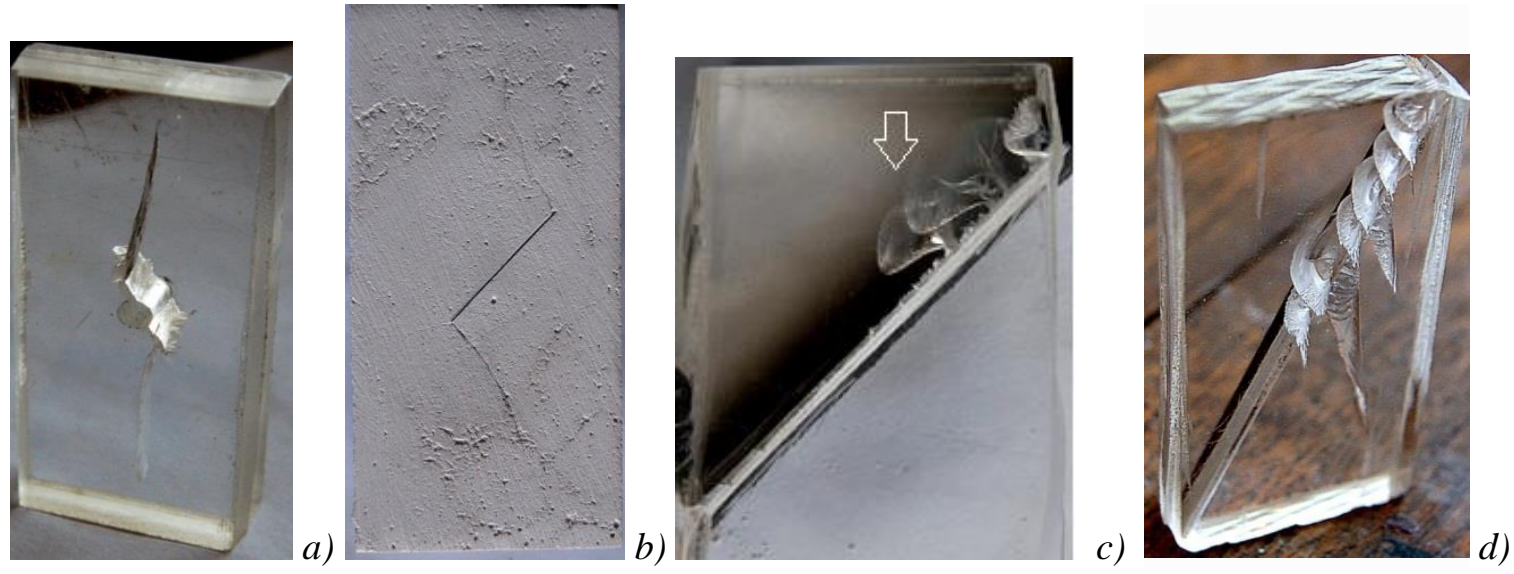

Fig. 3:a-Classical example of wing crack fracture in the neighborhood of an open cut (PMMA, slope angle of the cut is $30 \mathrm{deg}$. to the compression axis), $b$ - the wing cracks in a gypsum sample in the area of a smooth polymeric insert with low friction coefficient, $c$ - initial phase of feathering development at longitudinal shear, $d$ - a sample of an advanced phase of fracture.

In gypsum sample made with a plane polymeric insert and low friction coefficient on the surface of contact the fracture at compression begins with formation of a classical wing crack without secondary structures of fracture (Fig. 3b, sample dimensions (WxHxT): 50x100x24 mm, cut width $28 \mathrm{~mm}$, inclination 45 degrees, insert thickness $0.5 \mathrm{~mm}$ ). In case of a rough steel insert providing coupling with the contacting surface, the nature of fracture changes. Fracture develops on the continuation of the initial filled slit.

\section{Fracture under the influence of longitudinal shear}

In real materials the planes of sliding or analogues of the cuts are usually not a through the thickness defects. Therefore, at compression, in addition to system of wing cracks in end zone parts, they can initiate occurrence of feathering cracks on the lateral faces which are under the influence of stresses of longitudinal shear. The samples with a longitudinal cut, inclined in relation to the compression axis, and smooth steel plates densely inserted into it were axially compressed up to occurrence of feathering structures. The PMMA sample with feathering cracks at longitudinal shear is shown in Fig. 3c (sample dimensions (WxHxT): 37x61x12 mm, cut inclination 40 degrees), Fig. 3d (sample dimensions (WxHxT): $37 \times 61 \times 12 \mathrm{~mm}$, cut inclination 65 degrees). Development of the multiple regularly located feathering corresponds to the model of fracture development in the neighborhood of longitudinal shear suggested earlier (Goldstein, 2012). Note that the feathering was formed only on one side of the initial cut. 
Propagation of secondary faults on one side of initial shear plane was observed in experiments with one side cut and with one smooth insert. This can be related with the torque caused by the cut on one side of the sample. As long as secondary structures of feathering started from the edge of the cut (Fig. 3c) where the described effect has the maximum intensity, the fracture has shifted to one side of the cut.

\section{Conclusions}

The experiments confirm the possibility of wing-crack system formation (occurrence of wings) for two kinds of the preexisting defect. The first one is when there is an inclined surface of friction, including the rough one. The other one is when the site of slipping (and friction) is limited to the edges of the initial crack. However development of secondary structures of wing-crack in the specified variants was not realized in the experiments. The scenarios of complex wing-crack systems development seem to be more diverse, rather than it is given in the available theoretical models. For example, development of the symmetric system of feathering inside the initial shear of limited extent.

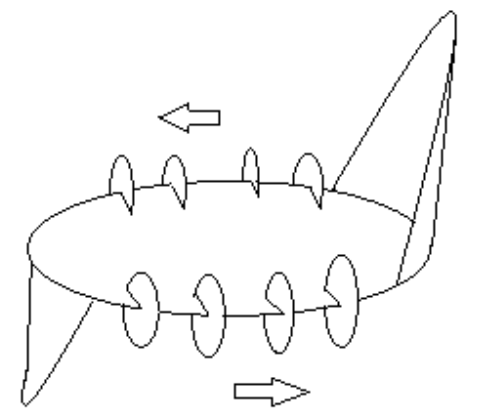

a)

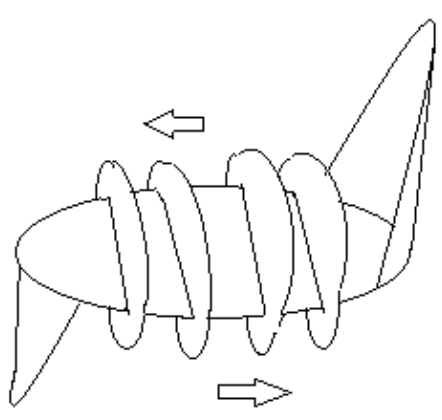

b)

Fig. 4: The schematic of wing-crack system containing along with the "wings", the periodic system of small ruptures of feathering on the periphery. The arrows follow shear direction

Let's remind that at compression of a body containing the inclined plane of shear (or a narrow cavity) the wing-crack system is formed containing, along with the "wings", the periodic system of small ruptures of feathering on the periphery of the area in which regard there is a longitudinal shear stress concentration (Fig. 4). Conditions of periodic system development on the extended front of longitudinal shear were discussed in (Goldstein, 2012).

\section{Acknowledgement}

This work was supported by the Russian Foundation for Basic Research (RFBR) under Grant No. 17-0100526.

\section{References}

Brogi A. (2011) Variation in fracture patterns in damage zones related to strike-slip faults interfering with preexisting fractures in sandstone (Calcione area, southern Tuscany, Italy) Journal of Structural Geology, 33, pp.644-661.

Germanovich, L.N., Salganik, R.L., Dyskin, A.V. and Lee, K.K (1994) Mechanisms of brittle fracture of rock with multiple pre-existing cracks in compression. Pure and Applied Geophysics, 143(13), pp. 117-149,

Germanovich, L.N. and Dyskin, A.V. (2000) Fracture mechanisms and instability of openings in compression. Int. J. Rock. Mech. Min. Sci., 37, pp. 263-284.

Goldstein, R.V. and Osipenko, N.M. (2012) Successive development of the structure of a fracture near the front of a longitudinal shear crack. Doklady Physics, 57(7), pp.281-284.

Moss, W.C. and Gupta, Y.M. (1982) A constitutive model describing dilatancy and cracking in brittle rocks. Journal of Geophysical Research, 87 (B4), pp. 2985-2998.

Zavattieri P. et al. (2011) Numerical Simulation of Crack Propagation in Rocks under Uniaxial Compression. Purdue University,Term Project CE-597, Non-linear fracture mechanics. 